\title{
Nonlinear Torsional Vibration Analysis and Nonlinear Feedback Control of Complex Permanent Magnet Semidirect Drive Cutting System in Coal Cutters
}

\author{
Lianchao Sheng, ${ }^{1,2}$ Wei Li $\mathbb{D}^{1},{ }^{1}$ Gaifang Xin, ${ }^{3}$ Yuqiao Wang $\left(\mathbb{D},{ }^{1}\right.$ \\ Mengbao Fan $\mathbb{D},{ }^{1}$ and Xuefeng Yang ${ }^{1}$ \\ ${ }^{1}$ School of Mechatronic Engineering, China University of Mining and Technology, Xuzhou 221116, China \\ ${ }^{2}$ School of Mechatronic Engineering, Jiangsu Normal University, Xuzhou 221116, China \\ ${ }^{3}$ School of Mechanical and Electrical Engineering, Changzhou College of Information Technology, Changzhou 213164, China
}

Correspondence should be addressed to Wei Li; cmeecumt512@yahoo.com

Received 16 October 2018; Revised 12 March 2019; Accepted 10 April 2019; Published 20 May 2019

Academic Editor: Marcelo Messias

Copyright (c) 2019 Lianchao Sheng et al. This is an open access article distributed under the Creative Commons Attribution License, which permits unrestricted use, distribution, and reproduction in any medium, provided the original work is properly cited.

\begin{abstract}
The semidirect drive cutting transmission system of coal cutters is prone to unstable torsional vibration when the resistance values of its driving permanent magnet synchronous motor (PMSM) are affected by changes in temperatures and tough conditions. Besides, the system has the properties of complex electromechanically coupling such as the coupling between electrical parameters and mechanical parameters. Therefore, in this study, the nonlinear torsional vibration equation was established on the basis of the Lagrange-Maxwell theory. Moreover, in light of the nonlinear dynamic bifurcation theory, the system stability was analyzed by taking the resistance value of power motor as the bifurcation parameter. In addition, the influence of subcritical bifurcation on the torsional vibration was studied by investigating the necessary and sufficient conditions for dynamic Hopf bifurcation and classifying the bifurcation types. At last, in order to suppress destabilizing oscillation induced by Hopf bifurcation, the nonlinear feedback controller was constructed, with the introduction of feedback from the motor velocity as well as the selection of voltage value on the $q$ shaft as the controlled variable. Meanwhile, the three-order normal form and controlling parameters of the system were obtained with the aid of the multiple scales method and the harmonic balance method. In this way, the Hopf bifurcation point was transferred to control the stability of Hopf bifurcation and the amplitude of limit cycle, thus guaranteeing reliable and safe operation of the system. The numerical simulation results indicate that the designed controller boosts an ideal controlling effect.
\end{abstract}

\section{Introduction}

When the tail drum of the permanent magnet semidirect drive cutting transmission system in the coal cutters cuts coals and rocks, due to the uneven intensity of the coal seam, the brittle caving of the coal rock mass, and hard parcels existing, the loads on the drum exhibit the characteristics of randomness, major fluctuations, great impact, etc. [13]. Besides, since coal cutters operates in a relatively tough environment, the electrical parameters of its driving motor change with the different temperatures. This not only causes abundant dynamic behaviors of the coupling nonlinear dynamic system, but also leads to the torsional vibration and instability phenomena of the system, thus affecting the working efficiency of coal cutters. The cutting transmission system of permanent magnet semidirect drive in coal cutters, which consists of a permanent magnet synchronous motor (PMSM), a speed reducer, cutting drums, and other components, is a typical electromechanical coupling system [46]. The electromechanical coupling mechanism and coupling dynamics are influenced by complicate factors, including mechanics, electromagnetism, and nonlinear dynamics [79]. The multivariable and multienergy-domain coupling existing within the complicate electromechanical coupling system is a basic characteristic which the system exhibits during nonstationary processes such as the starting, braking, and load variations. The electromechanical coupling vibration that happens in nonstationary processes will result in 
defective condition of the equipment and affect the dynamics and stability of the system. It may also give rise to major failure and even safety accidents. Therefore, it is essential to study electromechanical coupling dynamics of the semidirect drive cutting transmission system in coal cutters and the mechanism of torsional vibrations; thus the dynamics of the system will be further optimized.

At present, the research on the dynamic properties of cutting units in the coal cutter is mainly focused on the investigation of its whole vibration characteristics based on the virtual prototype technology $[10,11]$, the influence of gear clearance, meshing stiffness, and gear friction on the system dynamics [12, 13], and forces among gears [14, 15]. Scholars have conducted studies on system dynamics which take "motor-gear-actuator" as the model of transmission structure. The breakdown diagnosis $[16,17]$ and torsion vibration tests $[18,19]$ of the transmission system by means of dynamic analysis have become research hot spots. Taking the torsional vibration system of rolling mill with nonlinear friction damping as the research object, Liu et al. [20,21] analyzed the effects of both subcritical bifurcation and supercritical bifurcation on the system torsional vibration. With the introduction of the motor speed feedback, the nonlinear feedback controller was designed, in which the stator voltages of the drive motor were selected as the control variable, suppressing unstable vibrations for transmission system caused by Hopf bifurcation. The kinetic simulation technology was applied to one-stage gearbox and two-stage gearbox by W. Bartelmus [22], providing more ways for gearbox fault diagnosis. The transient dynamic response of drive motor-gear transmission system in starting process was investigated by $\mathrm{T}$. Khabou et al. [23]. Through the transfer matrix theory and the finite element analyses, Yu et al. [24] studied the propagation of torsional vibration in the shaft which was equipped with periodically attached local resonators. They were also the pioneers in finding the dispersion relation and incidental structure for such structure, confirming the existence of lowfrequency gap torsional vibration. Different load fluctuations may result in various rotational speed of the driving motor with the planetary gear. Depending on this case, F. Chaari et al. [25] investigated dynamic behaviors of the planetary gear by considering the fact that variable loads could induce a great change in rotational speed of the motor. Shi et al. $[26,27]$ illustrated the bifurcation structures and chaotic behaviors when the nonlinear torsional vibration system with nonlinear stiffness and nonlinear friction force was under the external excitation. The stability of undisturbed system was given by means of the stability theory of equilibrium positions of Hamiltonian systems. And chaotic behaviors under periodic disturbance were detected by using Melnikov theory. However, from the above, it can be seen that most public literatures attach high attention on the single aspect, either from mechanical perspective or from electrical aspect which cannot present a thorough insight into the mechanism of torsional vibration to provide the corresponding control methods. One reason to explain this limitation is the complex energy transmission between electromagnetic energy of the motor and kinetic energy of the machinery. Therefore, some scholars have started to study the reasons of torsional vibration and push for controlling methods from the view of electromechanical coupling. According to Xu et al. [28], the nonlinear electromechanically coupling dynamic equation of the driving system was given by the analysis of the nonlinear interactive magnetic forces in the electromechanical system in order to further study on the bifurcation and chaotic motions. The influence of the electromagnetic stiffness and the damping coefficient on the electromechanical system was generated by Tomasz Szolc et al. [29], where the rotating machine under dynamic coupling effect was modeled. Concerning those electromechanical coupling system of rolling mills with nonlinear friction force, the dynamic equation of its torsional vibration was set up by Liu et al. [30] through the adoption of electromechanical analytical dynamic method.

In this paper, the electromechanical coupling transmission system of coal cutters under the coupling effect of electrical parameters and mechanical parameters is studied, where the dynamic model of its nonlinear coupling torsional vibration is established with reference to the LagrangeMaxwell equation. Besides, based on numerical and analytical methods, the necessary and sufficient conditions are presented with various the stator resistance values of PMSM. Furthermore, the Hopf bifurcation types are determined, which makes it possible to analyze their influence on the torsional vibration of the system. Aimed at suppressing unstable vibration, the voltage value of the motor on the $\mathrm{q}$-axis is taken as control parameter, in which the velocity feedback is introduced; thus, the feedback controller under nonlinear states is designed. Finally, the methods of multiple scales and harmonic balance are employed to obtain the third-order normal form for the system and its controlling parameters, by which the bifurcation point is transferred, enhancing the system stability and decreasing the limit cycle amplitudes.

\section{Electromechanical Coupling Dynamic Model}

The permanent magnet semidirect drive cutting transmission system in coal cutters which incorporates a variety of subsystems serves as a typical complex electromechanical coupling system. The electromechanical coupling of such system mainly refers to the fact that the electromagnetic parameters of the PMSM are coupled with the mechanical parameters of the gear transmission system and the load system. The conversion of motor electrical energy requires air gap magnetic field. Unlike a three-phase asynchronous motor, which generates a magnetic field by passing current through a motor winding, a PMSM generates a magnetic field by a permanent magnet. In order to facilitate the establishment of an electromechanical coupling dynamics model, the latter part of the gear transmission system is equivalent to the end of the elastic connection shaft. The simplified model is shown in Figure 1.

Under the assumptions that (I) the core saturation, the eddy current, and hysteresis are ignored; (II) air gap distribution is uniform and the stator windings are star-connected; (III) no damping effect is on the rotor and the permanent 
TABLE 1: Generalized coordinates of electromechanical system.

\begin{tabular}{lccccc}
\hline Generalized coordinates & Stator & & Motor rotation angle & Mechanical system \\
& $j=1$ & $j=2$ & $j=3$ & $j=4$ & $j=5$ \\
\hline$q_{j}$ & $e_{a}$ & $e_{b}$ & $e_{c}$ & $\theta_{1}$ & $\theta_{2}$ \\
$\dot{q}_{j}$ & $i_{a}$ & $i_{b}$ & $i_{c}$ & $\dot{\theta}_{1}$ & $\dot{\theta}_{2}$ \\
$Q_{j}$ & $u_{a}$ & $u_{b}$ & $u_{c}$ & - & $T_{L}$ \\
\hline
\end{tabular}

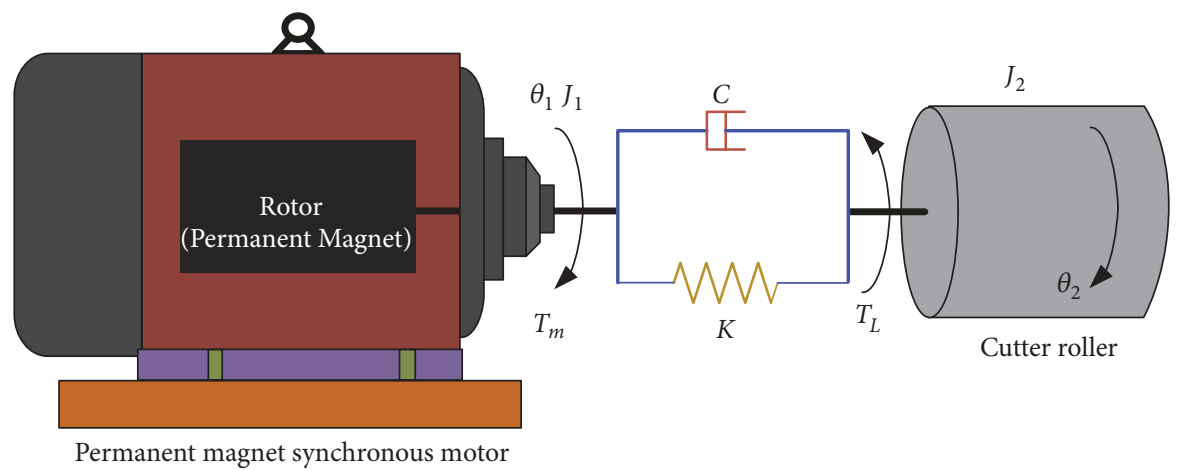

Figure 1: Schematic diagram of electromechanical coupling system in coal cutter.

magnet; (IV) the back electromotive force waveform is a sine wave, the dynamic equations of the system are established, as shown in the simplified model of Figure 1.

Based on the basic principles of electromechanical analysis dynamics and Lagrange-Maxwell equation, the mathematical model is established. The generalized coordinates of the system are first determined as shown in Table 1.

The specific calculation method of $T_{L}$ is shown in the literature [3]. The system kinetic energy $E$ is given by

$$
E=\frac{1}{2} J_{1} \dot{\theta}_{1}^{2}+\frac{1}{2} J_{2} \dot{\theta}_{2}^{2}
$$

$J_{1}$ is the moment of inertia of the rotor in the PMSM and $\mathrm{J}_{2}$ is the equivalent mass moment of inertia of the driven mechanical part reduced to the motor rotor rotation axis.

The system magnetic energy $W_{\mathrm{H}}$ is expressed as follows:

$$
\begin{gathered}
W_{H}=W_{H 1}+W_{H 2}=\left\{\frac{1}{2} L_{a} i_{a}^{2}+\frac{1}{2} L_{b} i_{b}^{2}+\frac{1}{2} L_{c} i_{c}^{2}\right. \\
\left.+H i_{a} i_{b}+H i_{b} i_{c}+H i_{a} i_{c}\right\}+\left\{i_{a} \psi_{f} \cos \theta_{1}\right. \\
\left.+i_{b} \psi_{f} \cos \left(\theta_{1}-\frac{2}{3} \pi\right)+i_{c} \psi_{f} \cos \left(\theta_{1}+\frac{2}{3} \pi\right)\right\}
\end{gathered}
$$

The magnetic energy $W_{\mathrm{H}}$ of the PMSM includes two parts: $W_{\mathrm{H} 1}$ is the magnetic energy generated by the stator current; $W_{\mathrm{H} 2}$ is the magnetic energy generated by stator current with the flux which is produced by rotor in the stator. $i_{a}, i_{\mathrm{b}}$, and $i_{\mathrm{c}}$ are the current at both ends of each winding. $L$ and $H$ are the self-inductance and mutual inductance of the stator threephase windings respectively. $\theta_{1}$ is the rotor position angle, and $\psi_{f}$ is the flux generated by the permanent magnet.
The system potential energy $V$ can be derived as follows:

$$
V=\frac{1}{2} k\left(\theta_{1}-\theta_{2}\right)^{2}
$$
follows:

The Lagrange function $L$ of the system is calculated as

$$
\begin{aligned}
L= & E+W_{H}-V=\frac{1}{2} J_{1} \dot{\theta}_{1}^{2}+\frac{1}{2} J_{2} \dot{\theta}_{2}^{2}+\left\{\frac{1}{2} L_{a} i_{a}^{2}\right. \\
& \left.+\frac{1}{2} L_{b} i_{b}^{2}+\frac{1}{2} L_{c} i_{c}^{2}+H i_{a} i_{b}+H i_{b} i_{c}+H i_{a} i_{c}\right\} \\
& +\left\{i_{a} \psi_{f} \cos \theta_{1}+i_{b} \psi_{f} \cos \left(\theta_{1}-\frac{2}{3} \pi\right)\right. \\
& \left.+i_{c} \psi_{f} \cos \left(\theta_{1}+\frac{2}{3} \pi\right)\right\}-\frac{1}{2} k\left(\theta_{1}-\theta_{2}\right)^{2}
\end{aligned}
$$
follows:

The system dissipation function $F$ can be expressed as

$$
\begin{aligned}
F & =F_{1}+F_{2} \\
& =\left\{\frac{1}{2} R_{a} i_{a}^{2}+\frac{1}{2} R_{b} i_{b}^{2}+\frac{1}{2} R_{c} i_{c}^{2}\right\}+\frac{1}{2} C\left(\dot{\theta}_{1}-\dot{\theta}_{2}\right)^{2}
\end{aligned}
$$

$F_{1}$ is the dissipation function of the electromagnetic system. $F_{2}$ is the dissipation function of the mechanical system. $R_{a}, R_{\mathrm{b}}$, and $R_{\mathrm{c}}$ are the three-phase resistance of the PMSM, and $R_{a}=R_{\mathrm{b}}=R_{\mathrm{c}}=R$. C is the viscous damping coefficients of the shaft.

The frictional resistance torque caused by the nonlinear friction damping at the end of semidirect drive transmission system in coal cutter takes a widely existing form of nonlinear sliding frictional torque.

$$
f\left(\dot{\theta}_{2}\right)=\kappa+\gamma \dot{\theta}_{2}+\chi \dot{\theta}_{2}^{2}
$$


Substituting (4) and (5) into the system's LagrangeMaxwell equation, the result is shown as follows:

$$
\frac{d}{d t}\left(\frac{\partial L}{\partial \dot{q}_{j}}\right)-\frac{\partial L}{\partial q_{j}}+\frac{\partial F}{\partial \dot{q}_{j}}=Q_{j}
$$

For the stator winding $A(j=1)$, the specific expansion form is shown as follows:

$$
\begin{aligned}
\frac{\partial L}{\partial q_{1}} & =0 \\
\frac{\partial L}{\partial \dot{q}_{1}} & =L i_{a}+H i_{b}+H i_{c}+\psi_{f} \cos \theta_{1} \\
\frac{d}{d t}\left(\frac{\partial L}{\partial \dot{q}_{1}}\right) & \longrightarrow \\
\frac{d}{d t}\left(\frac{\partial L}{\partial i_{a}}\right) & =L \frac{d i_{a}}{d t}+H \frac{d i_{b}}{d t}+H \frac{d i_{c}}{d t}+\frac{d}{d t}\left(\psi_{f} \cos \theta_{1}\right) \\
\frac{\partial F}{\partial \dot{q}_{1}} & \longrightarrow \frac{\partial F}{\partial i_{a}}=R_{a} i_{a} \\
Q_{1} & =\frac{d}{d t}\left(\frac{\partial L}{\partial \dot{q}_{1}}\right)-\frac{\partial L}{\partial q_{1}}+\frac{\partial F}{\partial \dot{q}_{1}}, \\
u_{a} & =Q_{1}
\end{aligned}
$$

So the voltage equation of the stator winding $A(j=1)$ can be expressed as follows:

$$
\begin{aligned}
u_{a} & =Q_{1}=\frac{d}{d t}\left(\frac{\partial L}{\partial \dot{q}_{1}}\right)-\frac{\partial L}{\partial q_{1}}+\frac{\partial F}{\partial \dot{q}_{1}} \\
& =L \frac{d i_{a}}{d t}+H \frac{d i_{b}}{d t}+H \frac{d i_{c}}{d t}+\frac{d}{d t}\left(\psi_{f} \cos \theta_{1}\right)+R_{a} i_{a}
\end{aligned}
$$

Similarly, the voltage equation of the stator winding $B(j=$ 2 ) is given by

$$
\begin{aligned}
u_{b}= & Q_{2}=\frac{d}{d t}\left(\frac{\partial L}{\partial \dot{q}_{2}}\right)-\frac{\partial L}{\partial q_{2}}+\frac{\partial F}{\partial \dot{q}_{2}} \\
= & L \frac{d i_{b}}{d t}+H \frac{d i_{a}}{d t}+M \frac{d i_{c}}{d t} \\
& +\frac{d}{d t}\left(\psi_{f} \cos \left(\theta_{1}-\frac{2}{3} \pi\right)\right)+R_{b} i_{b}
\end{aligned}
$$

The voltage equation of the stator winding $C(j=3)$ is given by

$$
\begin{aligned}
u_{c}= & Q_{3}=\frac{d}{d t}\left(\frac{\partial L}{\partial \dot{q}_{3}}\right)-\frac{\partial L}{\partial q_{3}}+\frac{\partial F}{\partial \dot{q}_{3}} \\
= & L \frac{d i_{c}}{d t}+H \frac{d i_{a}}{d t}+H \frac{d i_{b}}{d t} \\
& +\frac{d}{d t}\left(\psi_{f} \cos \left(\theta_{1}+\frac{2}{3} \pi\right)\right)+R_{c} i_{c}
\end{aligned}
$$

The motion equation of the motor rotation angle $(j=4)$ is formulated,

$$
\begin{aligned}
J_{1} \frac{d \dot{\theta}_{1}}{d t}= & -i_{a} \psi_{f} \sin \theta_{1}-i_{b} \psi_{f} \sin \left(\theta_{1}-\frac{2}{3} \pi\right) \\
& -i_{c} \psi_{f} \sin \left(\theta_{1}+\frac{2}{3} \pi\right)-k\left(\theta_{1}-\theta_{2}\right) \\
& -C\left(\dot{\theta}_{1}-\dot{\theta}_{2}\right)
\end{aligned}
$$

The motion equation of the mechanical system $(j=5)$ is generated,

$$
\begin{aligned}
J_{2} \frac{d \dot{\theta}_{2}}{d t}= & -k\left(\theta_{1}-\theta_{2}\right)+C\left(\dot{\theta}_{1}-\dot{\theta}_{2}\right)+T_{L}+\kappa+\gamma \dot{\theta}_{2} \\
& +\chi \dot{\theta}_{2}^{2}
\end{aligned}
$$

The mathematical model of PMSM in $A B C$ three-phase stationary coordinate system is shown in the following equation:

$$
\begin{aligned}
& {\left[\begin{array}{l}
u_{a} \\
u_{b} \\
u_{c}
\end{array}\right]=} {\left[\begin{array}{lll}
R & 0 & 0 \\
0 & R & 0 \\
0 & 0 & R
\end{array}\right]\left[\begin{array}{l}
i_{a} \\
i_{b} \\
i_{c}
\end{array}\right]+\frac{d}{d t}\left[\begin{array}{ccc}
L & H & H \\
H & L & H \\
H & H & L
\end{array}\right]\left[\begin{array}{l}
i_{a} \\
i_{b} \\
i_{c}
\end{array}\right] } \\
&+\frac{d}{d t}\left[\begin{array}{c}
\psi_{f} \cos \theta_{1} \\
\psi_{f} \cos \left(\theta_{1}-\frac{2}{3} \pi\right) \\
\psi_{f} \cos \left(\theta_{1}+\frac{2}{3} \pi\right)
\end{array}\right]
\end{aligned}
$$

The system dynamics equation can be written as follows:

$$
\begin{aligned}
& L_{a} \frac{d i_{a}}{d t}+H \frac{d i_{b}}{d t}+H \frac{d i_{c}}{d t}-\psi_{f} \dot{\theta}_{1} \sin \theta_{1}+R i_{a}=u_{a} \\
& L_{b} \frac{d i_{b}}{d t}+H \frac{d i_{a}}{d t}+H \frac{d i_{c}}{d t}-\psi_{f} \dot{\theta}_{1} \sin \left(\theta_{1}-\frac{2}{3} \pi\right)+R i_{b} \\
& \quad=u_{b} \\
& L_{c} \frac{d i_{c}}{d t}+H \frac{d i_{a}}{d t}+H \frac{d i_{b}}{d t}-\psi_{f} \dot{\theta}_{1} \sin \left(\theta_{1}+\frac{2}{3} \pi\right)+R i_{c} \\
& =u_{c} \\
& J_{1} \ddot{\theta}_{1}+C\left(\dot{\theta}_{1}-\dot{\theta}_{2}\right)+K\left(\theta_{1}-\theta_{2}\right) \\
& =-i_{a} \psi_{f} \sin \theta_{1}-i_{b} \psi_{f} \sin \left(\theta_{1}-\frac{2}{3} \pi\right) \\
& \quad-i_{c} \psi_{f} \sin \left(\theta_{1}+\frac{2}{3} \pi\right) \\
& J_{2} \ddot{\theta}_{2}-C\left(\dot{\theta}_{1}-\dot{\theta}_{2}\right)-K\left(\theta_{1}-\theta_{2}\right) \\
& =T_{L}+\kappa+\gamma \dot{\theta}_{2}+\chi \dot{\theta}_{2}^{2}
\end{aligned}
$$

From (15), as a variable-coefficient differential equation, the established mathematical model is more complex. In 


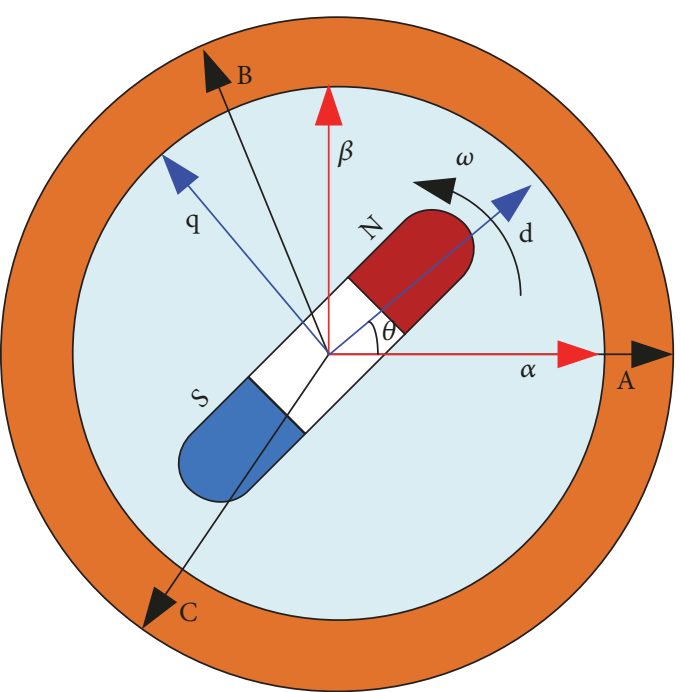

FIGURE 2: Principle of coordinate transformation.

order to simplify the equation and describe the motion state conveniently, the $A B C$ three-phase stationary coordinate system is converted to the $d q$ axis coordinate system.

The principle of coordinate conversion is shown in Figure 2. Firstly, the $A B C$ three-phase coordinate system needs to be converted to the $\alpha \beta$ plane stationary coordinate system. Then, the $\alpha \beta$ plane stationary coordinate system is converted to the $d q$ axis coordinate system. Finally the coordinate conversion equation is written as follows:

$$
\begin{aligned}
& C_{3 s / 2 r} \\
& =\sqrt{\frac{2}{3}}\left[\begin{array}{ccc}
\cos \theta & \cos \left(\theta-\frac{2 \pi}{3}\right) & \cos \left(\theta+\frac{2 \pi}{3}\right) \\
-\sin \theta & -\sin \left(\theta-\frac{2 \pi}{3}\right) & -\sin \left(\theta+\frac{2 \pi}{3}\right) \\
\sqrt{\frac{1}{2}} & \sqrt{\frac{1}{2}} & \sqrt{\frac{1}{2}}
\end{array}\right]
\end{aligned}
$$

The electromechanical coupling dynamic equation of the system is shown as follows:

$$
\begin{aligned}
& \dot{I}_{d}=-\frac{R}{L-H} I_{d}+\frac{u_{d}}{L-H}+\omega_{1} I_{q} \\
& \dot{I}_{q}=-\frac{R}{L-H} I_{q}+\frac{u_{q}}{L-H}-\frac{\sqrt{6} \psi_{f}}{2(L-H)} \omega_{1}-\omega_{1} I_{d} \\
& \dot{\alpha}=\omega_{1}-\omega_{2} \\
& \dot{\omega}_{1}=\frac{1}{J_{1}}\left[-C\left(\omega_{1}-\omega_{2}\right)-K \alpha-\frac{\sqrt{6} \psi_{f}}{2} I_{q}\right] \\
& \dot{\omega}_{2}=\frac{1}{J_{2}}\left[C\left(\omega_{1}-\omega_{2}\right)+K \alpha+\kappa+\gamma \omega_{2}+\chi \omega_{2}^{2}+T_{L}\right]
\end{aligned}
$$

\section{Hopf Bifurcation Analysis of Electromechanical Coupling System}

Taking $k_{1}=-R /(L-H), k_{2}=-\sqrt{6} \psi_{f} / 2(L-H)$, and $k_{3}=-\sqrt{6} \psi_{f} / 2 J_{1},\left(\begin{array}{lllll}I_{d} & I_{q} & \alpha & \omega_{1} & \omega_{2}\end{array}\right)^{T}$ is expressed with $\left(\begin{array}{lllll}x_{1} & x_{2} & x_{3} & x_{4} & x_{5}\end{array}\right)^{T}$. Then the equilibrium point of (17) can be transferred to the origin of coordinates by proper linear transformation. Without loss of generality, the dynamic characteristics of the equilibrium point of the system at the origin are studied, and the Jacobian matrix of the system at the origin can be expressed as follows:

$$
\boldsymbol{A}(0, R)=\left(\begin{array}{ccccc}
k_{1} & 0 & 0 & 0 & 0 \\
0 & k_{1} & 0 & k_{2} & 0 \\
0 & 0 & 0 & 1 & -1 \\
0 & k_{3} & -\frac{K}{J_{1}} & -\frac{C}{J_{1}} & \frac{C}{J_{1}} \\
0 & 0 & \frac{K}{J_{2}} & \frac{C}{J_{2}} & \frac{\gamma-C}{J_{2}}
\end{array}\right)
$$

The stability of the system at the equilibrium point is determined by the real part of the eigenvalues of the Jacobian matrix $\boldsymbol{A}(0, R)$. If the eigenvalues of Jacobian matrix $\boldsymbol{A}(0, R)$ are all negative, then the system is asymptotically stable at the equilibrium point; If there is a positive real part in the eigenvalues of $\boldsymbol{A}(0, R)$, then the system is unstable at the equilibrium point and easily induces instability oscillation; if there is a pure imaginary root in the eigenvalue of $\boldsymbol{A}(0, R)$, then the higher order terms need to be analyzed. At this time, the system has rich dynamic behavior.

The characteristic equation of the system Jacobian matrix $\boldsymbol{A}(0, R)$ can be expressed as follows:

$$
\lambda^{5}+p_{1} \lambda^{4}+p_{2} \lambda^{3}+p_{3} \lambda^{2}+p_{4} \lambda+p_{5}=0
$$

In (19),

$p_{1}=\frac{C}{J_{1}}+\frac{C-\gamma}{J_{2}}-2 k_{1}$

$p_{2}$

$$
\begin{aligned}
= & \frac{K+J_{2} k_{1}^{2}+2 \gamma k_{1}-2 C k_{1}-J_{2} k_{2} k_{3}}{J_{2}}+\frac{K-2 k_{1} C}{J_{1}} \\
& -\frac{\gamma C}{J_{1} J_{2}}
\end{aligned}
$$

$p_{3}$

$$
\begin{aligned}
= & \frac{C k_{1}^{2}-2 K k_{1}}{J_{1}} \\
& +\frac{C k_{1}^{2}+J_{2} k_{1} k_{2} k_{3}-\gamma k_{1}^{2}-2 K k_{1}-(C-\gamma) k_{2} k_{3}}{J_{2}} \\
& +\frac{2 k_{1} \gamma C-\gamma K}{J_{1} J_{2}},
\end{aligned}
$$


$p_{4}$

$$
\begin{aligned}
= & \frac{K k_{1}^{2}}{J_{1}}+\frac{K k_{1}^{2}+(C-\gamma) k_{1} k_{2} k_{3}-K k_{2} k_{3}}{J_{2}} \\
& +\frac{2 K k_{1} \gamma-k_{1}^{2} \gamma C}{J_{1} J_{2}}, \\
p_{5}= & \frac{K k_{1} k_{2} k_{3}}{J_{2}}-\frac{\gamma K k_{1}^{2}}{J_{1} J_{2}}
\end{aligned}
$$

The Hurwitz determinant can be constituted by the coefficient $p_{i}$ and be expressed in the following form.

$$
\Delta_{i}=\left|\begin{array}{ccccc}
p_{1} & 1 & 0 & \cdots & 0 \\
p_{3} & p_{2} & p_{1} & \cdots & 0 \\
p_{5} & p_{4} & p_{3} & \cdots & p_{1} \\
\vdots & \vdots & \vdots & & \vdots \\
p_{2 i-1} & p_{2 i-2} & p_{2 i-3} & \cdots & p_{i}
\end{array}\right|
$$

If $i>5$, then $p_{i}=0 . R=R_{0}$ is defined as the Hopf bifurcation point of the original system. According to the Hurwitz determinant, the necessary and sufficient conditions for Hopf bifurcation in the system can be expressed as follows:

$$
\begin{array}{rl}
p_{i}\left(R_{0}\right)>0 & i=1,2,3, \ldots, 5 \\
\Delta_{i}\left(R_{0}\right)>0 & i=1,2,3 \\
\Delta_{4}\left(R_{0}\right)=0 & \\
\left.\frac{d\left(\Delta_{4}(R)\right)}{d R}\right|_{R=R_{0}} \neq 0 &
\end{array}
$$

Let the pure imaginary root of the system be $\pm \omega_{0} i$. The normalized left and right characteristic vectors of the matrix $A(0, \mathrm{R})$ corresponding to the eigenvalue $\omega_{0} i$ are denoted by $U$ and $V$, respectively.

$$
\begin{aligned}
& U A=\omega_{0} i U \\
& A V=\omega_{0} i V \\
& U V=1
\end{aligned}
$$

Let

$$
\begin{aligned}
\Psi & =\operatorname{Re}\left(-U f_{x x x} \boldsymbol{V} \boldsymbol{V} \boldsymbol{V}^{*}+2 U f_{x x} \boldsymbol{V} A^{-1}(0) f_{x x} \boldsymbol{V} \boldsymbol{V}^{*}\right. \\
& \left.+U f_{x x} \boldsymbol{V}^{*} \times\left[\boldsymbol{A}(0)-2 i \omega_{0} I\right]^{-1} f_{x x} \boldsymbol{V} \boldsymbol{V}\right)
\end{aligned}
$$

$\boldsymbol{V}^{*}$ is the conjugate complex number of $\boldsymbol{V}$.

$$
\begin{aligned}
& \boldsymbol{A}(0)=\left.\boldsymbol{A}(0, R)\right|_{R=R_{0}}, \\
& f_{x x x} \boldsymbol{V} \boldsymbol{V} \boldsymbol{V}^{*}=\frac{\partial}{\partial x}\left(\left(\frac{\partial}{\partial x}\left(\left(\frac{\partial f(x, R)}{\partial x}\right) \times \boldsymbol{V}\right) \times \boldsymbol{V}\right)\right. \\
& \left.\times \boldsymbol{V}^{*}\right)\left.\right|_{R=R_{0}, x=0} \cdot
\end{aligned}
$$

According to the positive or negative values of $\Psi$, the stability of the periodic solution during the Hopf bifurcation period can be determined. If $\Psi>0$ the bifurcation period of the system is asymptotically stable, and the limit cycle is stable. The Hopf bifurcation occurring at this time is supercritical bifurcation. If $\Psi<0$, the bifurcation period of the system is unstable, and the limit cycle is unstable. The Hopf bifurcation occurring at this time is subcritical bifurcation.

The mechanical and electrical parameters of the permanent magnet semidirect drive system in the coal cutter are taken as follows: $L=2.3 \times 10^{\wedge}-3 \mathrm{H}, H=6.2 \times 10^{\wedge}-5 \mathrm{H}$, $K=400000 \mathrm{~N} \cdot \mathrm{m} / \mathrm{rad}, J_{1}=J_{2}=100 \mathrm{~kg} \cdot \mathrm{m}^{2} \cdot \psi_{f}=3.8 \mathrm{~Wb}$. Numerical simulation analysis of system model is carried out in Matlab2015b. The numerical simulation results show that when $R=R_{0}=0.09 \Omega$, Hopf bifurcation occurs in the system, which could cause system instability and oscillation. Substituting $R_{0}$ into (24), $\Psi=-0.8962<0$, so the periodic orbit is unstable and the Hopf bifurcation is a complex subcritical bifurcation.

With different resistance values of the PMSM in the neighborhood of the subcritical Hopf bifurcation point, the motion state of the input elastic torque shaft of permanent magnet semidirect drive electromechanical coupling system is shown as Figures $3-6$. When $R=0.1 \Omega$ which is away from the limit $R_{0}=0.09 \Omega$ of instability, the motion state of the input elastic torque shaft is stable and is shown as Figure 3. When $R=0.094 \Omega$ which is near the limit $R_{0}=0.09 \Omega$ of instability, there are two different trajectories determined by the initial conditions. When the initial value is closer to the equilibrium point, taking $\left[\begin{array}{lllll}0.1 & 0.1 & 0.1 & 0.1 & 0.1\end{array}\right]$, the motion state of the input elastic torque shaft is stable and is shown as Figure 4; when the initial point is farther from the initial point, taking $\left[\begin{array}{lllll}0.7 & 0.7 & 0.7 & 0.7 & 0.7\end{array}\right]$, the motion state of the input elastic torque shaft is unstable and is shown as Figure 5. The amplitude of limit cycle is large. The system becomes unstable which could cause system instability oscillation. When $R$ is less than $R_{0}$, for any initial condition, the motion state of the input elastic torque shaft is divergent and unstable. Taking $R=0.085 \Omega$ as an instance, the system is in a diverging state and as Figure 6.

It can be found through the above analysis that when the system makes subcritical bifurcation, the behavior of the system is more complicated. Under normal circumstances, the initial conditions will have an important impact on the stability of the system. When the motor resistance $R$ is close to or smaller than the Hopf bifurcation point, a large amplitude unstable oscillation will burst, and the system will suddenly burst from stable to unstable. Therefore, when subcritical Hopf bifurcation occurs in the system, a sudden violent torsional oscillation will be caused on the input elastic torque shaft of the permanent magnet semidirect drive electromechanical coupling system in coal cutter, which will do harm to the safe and reliable operation of the system. In order to avoid this phenomenon, it is necessary to propose a control method for Hopf bifurcation in the above system and suppress the occurrence of subcritical bifurcation by shifting the bifurcation point or reducing the amplitude of vibration to avoid the occurrence of destructive oscillations. 


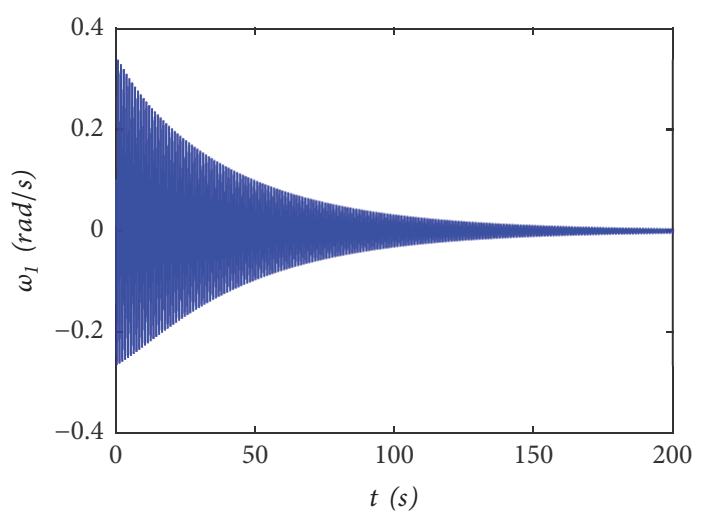

(a)

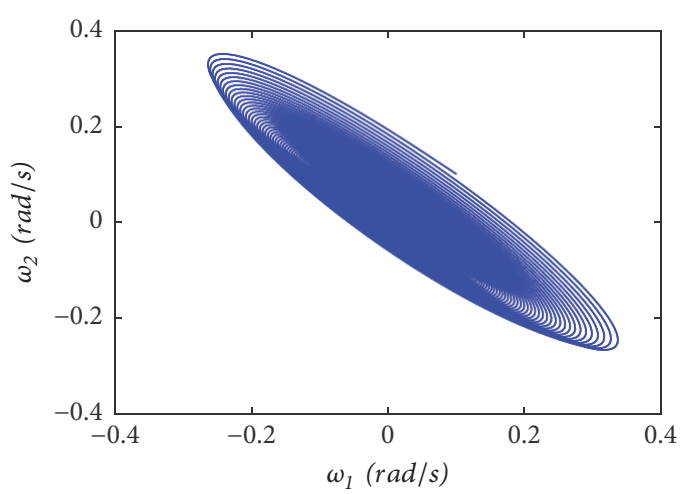

(b)

FIGURE 3: When the motor resistance $R=0.1 \Omega$, initial value $=\left[\begin{array}{lllll}0.1 & 0.1 & 0.1 & 0.1 & 0.1\end{array}\right]$, system motion state: (a) time history diagram; (b) phase diagram.

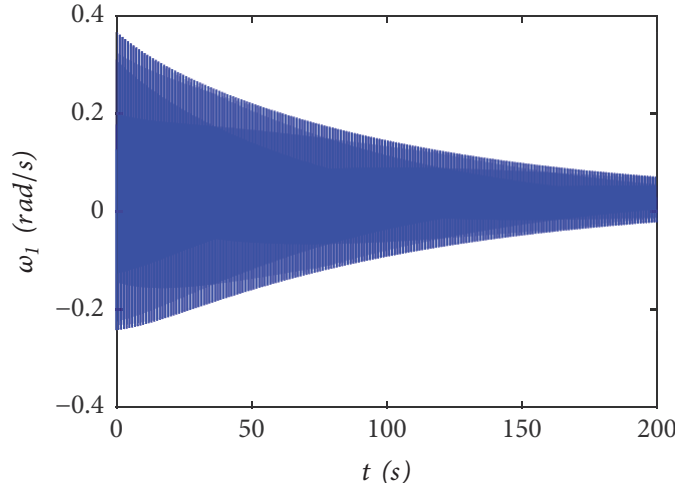

(a)

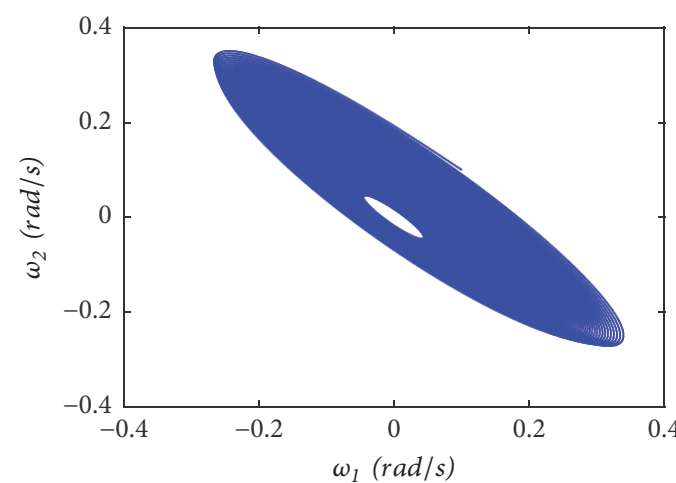

(b)

FIGURE 4: When the motor resistance $R=0.094 \Omega$, initial value $=\left[\begin{array}{lllll}0.1 & 0.1 & 0.1 & 0.1 & 0.1\end{array}\right]$, system motion state: (a) time history diagram; (b) phase diagram.

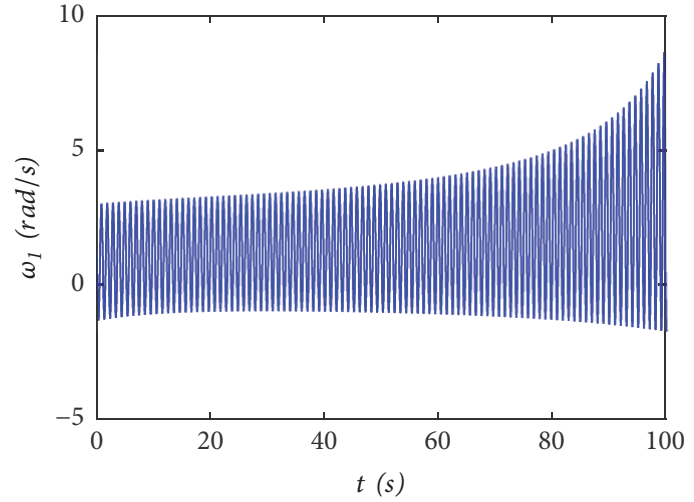

(a)

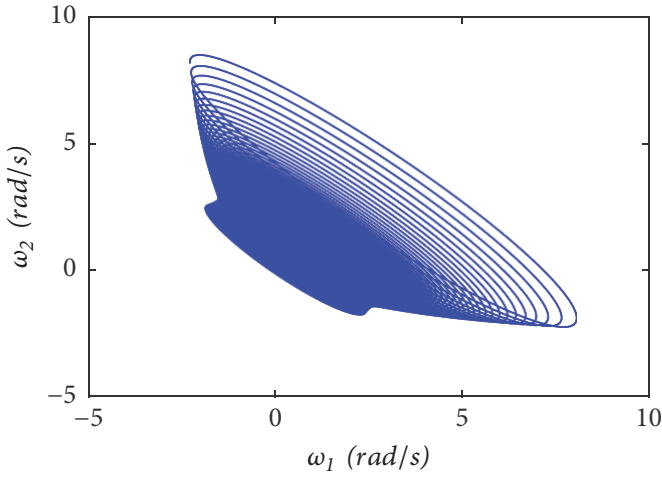

(b)

FIGURE 5: When the motor resistance $R=0.094 \Omega$, initial value $=\left[\begin{array}{lllll}0.7 & 0.7 & 0.7 & 0.7 & 0.7\end{array}\right]$, system motion state: (a) time history diagram; (b) phase diagram.

\section{Nonlinear Feedback Control of Electromechanical Coupling System}

The vector control method with of $I_{\mathrm{d}}=0$ is adopted, and the $\mathrm{q}$-axis voltage of the PMSM stands for a controlled quantity. A nonlinear state feedback controller is constructed as follows by introducing the motor speed feedback.

$$
u_{q}=K_{c} \omega_{1}+K_{n} \omega_{1}^{3}
$$




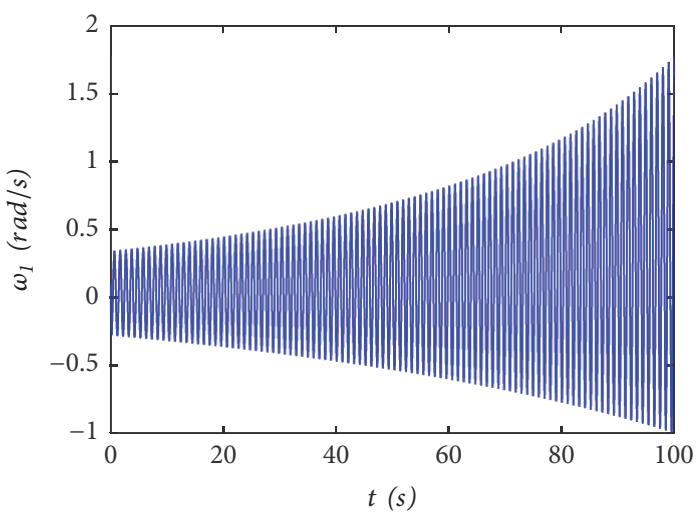

(a)

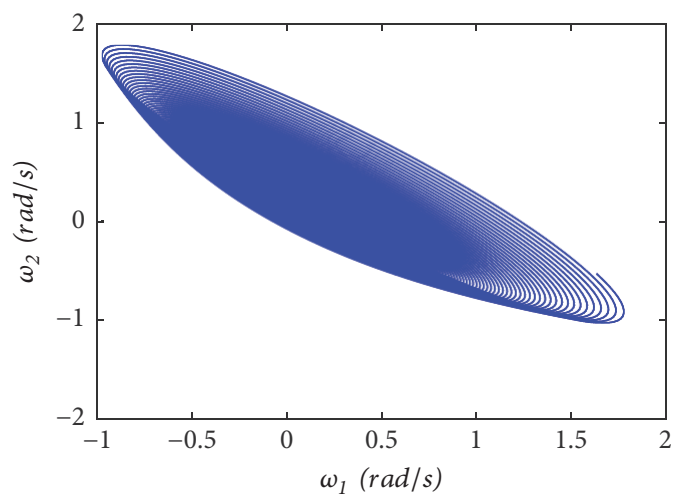

(b)

FIGURE 6: When the motor resistance $R=0.085 \Omega$, initial value $=\left[\begin{array}{lllll}0.1 & 0.1 & 0.1 & 0.1 & 0.1\end{array}\right]$, system motion status: (a) time history diagram; (b) phase diagram.

$K_{c}$ is a linear state feedback parameter. $K_{n}$ is a nonlinear state feedback parameter.

Since the vector control method with $I_{\mathrm{d}}=0$ is used, the bifurcation characteristics is no longer affected by the first equation of the system. The system state equation can be written as follows:

$$
\begin{aligned}
& \dot{x}_{2}=-\frac{R}{L-H} x_{2}+\frac{K_{c} x_{4}+K_{n} x_{4}^{3}}{L-H}-\frac{\sqrt{6} \psi_{f}}{2(L-H)} x_{4} \\
& \dot{x}_{3}=x_{4}-x_{5} \\
& \dot{x}_{4}=\frac{1}{J_{1}}\left[-C\left(x_{4}-x_{5}\right)-K x_{3}-\frac{\sqrt{6} \psi_{f}}{2} x_{2}\right] \\
& \dot{x}_{5}=\frac{1}{J_{2}}\left[C\left(x_{4}-x_{5}\right)+K x_{3}+\kappa+\gamma x_{5}+\chi x_{5}^{2}+T_{L}\right]
\end{aligned}
$$

According to the state equation, it can be found that the equilibrium point of the system remains unchanged after adding the nonlinear state feedback controller.

Let $k_{c}=K_{c} /(L-H)$; let the Jacobian matrix of the system be expressed as follows:

$$
A^{\prime}(0, R)=\left(\begin{array}{cccc}
k_{1} & 0 & k_{c}+k_{2} & 0 \\
0 & 0 & 1 & -1 \\
k_{3} & -\frac{K}{J_{1}} & -\frac{C}{J_{1}} & \frac{C}{J_{1}} \\
0 & \frac{K}{J_{2}} & \frac{C}{J_{2}} & \frac{\gamma-C}{J_{2}}
\end{array}\right)
$$

The characteristic equation of the Jacobian matrix is developed as

$$
\lambda^{4}+p_{1}^{\prime} \lambda^{3}+p_{2}^{\prime} \lambda^{2}+p_{3}^{\prime} \lambda+p_{4}^{\prime}=0
$$

In (29),

$$
\begin{aligned}
p_{1}^{\prime}= & \frac{C}{J_{1}}+\frac{C-\gamma}{J_{2}}-k_{1}, \\
p_{2}^{\prime}= & \frac{K-C k_{1}}{J_{1}}+\frac{K-C k_{1}+k_{1} \gamma}{J_{2}}-\frac{C \gamma}{J_{1} J_{2}} \\
& -\left(k_{2} k_{3}+k_{3} k_{c}\right) \\
p_{3}^{\prime}= & \frac{-K k_{1}}{J_{1}} \\
& +\frac{k_{2} k_{3} \gamma+k_{c} k_{3} \gamma-K k_{1}-C k_{2} k_{3}-C k_{3} k_{c}}{J_{2}} \\
& +\frac{C k_{1} \gamma-K \gamma}{J_{1} J_{2}}, \\
p_{4}^{\prime}= & \frac{-K k_{2} k_{3}-K k_{3} k_{c}}{J_{2}}+\frac{K k_{1} \gamma}{J_{1} J_{2}}
\end{aligned}
$$

The Hurwitz determinant is constituted by the characteristic equation coefficient $p_{i}^{\prime}$ and shown as follows:

$$
\Delta_{i}^{\prime}=\left|\begin{array}{ccccc}
p_{1}^{\prime} & 1 & 0 & \cdots & 0 \\
p_{3}^{\prime} & p_{2}^{\prime} & p_{1}^{\prime} & \cdots & 0 \\
p_{5}^{\prime} & p_{4}^{\prime} & p_{3}^{\prime} & \cdots & p_{1}^{\prime} \\
\vdots & \vdots & \vdots & & \vdots \\
p_{2 i-1}^{\prime} & p_{2 i-2}^{\prime} & p_{2 i-3}^{\prime} & \cdots & p_{i}^{\prime}
\end{array}\right|
$$

If $i>4$, then $p_{i}^{\prime}=0 . R=R_{0}^{*}$ is defined as the Hopf bifurcation point of the original system. The necessary and 
sufficient conditions for Hopf bifurcation in the system can be expressed in terms of the Hurwitz determinant,

$$
\begin{array}{rl}
p_{i}^{\prime}\left(R_{0}^{*}\right)>0 & i=1,2,3,4 \\
\Delta_{i}^{\prime}\left(R_{0}^{*}\right)>0 & i=1,2 \\
\Delta_{4}^{\prime}\left(R_{0}^{*}\right)=0 & \\
\left.\frac{d\left(\Delta_{4}^{\prime}(R)\right)}{d R}\right|_{R=R_{0}^{*}} \neq 0 &
\end{array}
$$

According to the calculation results of the system Hurwitz determinant, it is found that the Hopf bifurcation point can be transferred with different values of the parameter $K_{c}$ for a larger stable interval. To be more specific, when $K_{\mathrm{c}}=0.5$, the bifurcation point is transferred to $R_{0}^{*}=0.079 \Omega$, then the minimum value of the stable area of the system changes from 0.09 to 0.079 ; when $K_{\mathrm{c}}=1$, the bifurcation point is transferred to $R_{0}^{*}=0.0679 \Omega$, and the minimum value of the stable area of the system reduces to 0.0679 from 0.09 ; when $K_{\mathrm{c}}=1.5$, the bifurcation point is transferred to $R_{0}^{*}=0.0565 \Omega$, and the minimum value of the stable area of the system decreases from 0.09 to 0.0565 . According to the stable interval corresponding to different $K_{\mathrm{c}}$ values, it can be observed that the stable area of the controlled system is significantly larger than the original system. And when the system undergoes different temperatures, the stable area of the permanent magnet semidirect drive electromechanical coupling transmission system in coal cutter will expand.

For a better understanding on system's Hopf bifurcation, nonlinear analysis is performed on the system to realize the stability control of the Hopf bifurcation periodic solution and the amplitude control of the limit cycle. Considering subcritical bifurcation point $R_{0}^{*}=0.0565 \Omega$ and $K_{\mathrm{c}}=1.5$ as an instance, let $R=R_{0}^{*}+\eta, \eta$ is the perturbation amount of the bifurcation parameter $R_{0}^{*}$. When the perturbation quantity $\eta=0$, the eigenvalues of the system Jacobian matrix are $\lambda_{1,2}= \pm 6.1268 i, \lambda_{3}=-26.4589$, and $\lambda_{4}=-0.0056$, and the eigenvectors corresponding to the eigenvalue are $(\mathbf{u}+\mathbf{v i}, \mathbf{u}-$ vi, $\mathbf{U}_{1}, \mathbf{U}_{2}$ ). The system transformation matrix can be written as $\mathbf{T}=\left[\begin{array}{llll}\mathbf{u} & \mathbf{v} & \mathbf{U}_{1} & \mathbf{U}_{2}\end{array}\right]$. The following linear transformation is performed for (27):

$$
\mathbf{X}=\mathbf{T Y}, \quad \mathbf{Y}=\left[\begin{array}{llll}
y_{1} & y_{2} & y_{3} & y_{4}
\end{array}\right]^{T}
$$

The nonlinear standard equation of the system at the critical point can be expressed as follows:

$$
\dot{\mathbf{Y}}=\mathbf{J Y}+h(\mathbf{Y})
$$

$\boldsymbol{J}$ is the Jordan standard form of the linearization matrix of the nonlinear rotation system. $\boldsymbol{h}(x)$ is a nonlinear term.

$$
\begin{aligned}
& \boldsymbol{J}=\left(\begin{array}{cccc}
0 & \omega_{0} & 0 & 0 \\
-\omega_{0} & 0 & 0 & 0 \\
0 & 0 & -\alpha_{3} & 0 \\
0 & 0 & 0 & -\alpha_{4}
\end{array}\right) \\
& \boldsymbol{h}(x)=\left(\begin{array}{c}
0.0151 k_{n}\left(\begin{array}{l}
-0.0179 y_{1}-0.0043 y_{2} \\
+0.0009 y_{3}-0.0179 y_{4}
\end{array}\right)^{3}+26.8519\left(\begin{array}{c}
\kappa+T_{L} \\
+\chi\left(0.0155 y_{1}+0.0114 y_{2}-0.0179 y_{4}\right)^{2}
\end{array}\right) \\
0.0183 k_{n}\left(\begin{array}{c}
-0.0179 y_{1}-0.0043 y_{2} \\
+0.0009 y_{3}-0.0179 y_{4}
\end{array}\right)^{3}+6.6261\left(\begin{array}{c}
\kappa+T_{L} \\
+\chi\left(0.0155 y_{1}+0.0114 y_{2}-0.0179 y_{4}\right)^{2}
\end{array}\right) \\
0.9587 k_{n}\left(\begin{array}{c}
-0.0179 y_{1}-0.0043 y_{2} \\
+0.0009 y_{3}-0.0179 y_{4}
\end{array}\right)^{3}+1.15396\left(\begin{array}{c}
\kappa+T_{L} \\
+\chi\left(0.0155 y_{1}+0.0114 y_{2}-0.0179 y_{4}\right)^{2}
\end{array}\right) \\
0.0262 k_{n}\left(\begin{array}{c}
-0.0179 y_{1}-0.0043 y_{2} \\
+0.0009 y_{3}-0.0179 y_{4}
\end{array}\right)^{3}-28.3907\left(\begin{array}{c}
\kappa \\
+\chi\left(0.0155 y_{1}+0.0114 y_{2}-0.0179 y_{4}\right)^{2}
\end{array}\right)
\end{array}\right)
\end{aligned}
$$

Equation (34) can be expanded as follows:

$$
\begin{aligned}
& \dot{y}_{1}=\omega_{0} y_{2}+h_{1}(y) \\
& \dot{y}_{2}=-\omega_{0} y_{1}+h_{2}(y) \\
& \dot{y}_{3}=-\alpha_{3} y_{3}+h_{3}(y) \\
& \dot{y}_{4}=-\alpha_{4} y_{4}+h_{4}(y)
\end{aligned}
$$

From (37) (39) and on the basis of the method of multiple scales, the normal form of the nonlinear system can be calculated by introducing the following new variables:

$$
T_{k}=\varepsilon^{k} t \quad \mathrm{k}=0,1,2, \ldots
$$

That is, $T_{0}=t, T_{1}=\varepsilon t, T_{2}=\varepsilon^{2} t, \ldots \varepsilon$ is a small parameter. So the derivative to $t$ becomes an expanded form of the partial derivative to $T_{k}$. 


$$
\begin{aligned}
\frac{d}{d t} & =\frac{d T_{0}}{d t} \frac{\partial}{\partial T_{0}}+\frac{d T_{1}}{d t} \frac{\partial}{\partial T_{1}}+\cdots=D_{0}+\varepsilon D_{1}+\cdots \\
\frac{d^{2}}{d t^{2}} & =D_{0}^{2}+2 \varepsilon D_{0} D_{1}+\varepsilon^{2}\left(D_{1}^{2}+2 D_{0} D_{2}\right)+\cdots
\end{aligned}
$$

$D_{i}$ denotes $\partial / \partial T_{i}$, the solution of the equation can be expressed as follows,

$$
\begin{aligned}
y_{i}(t, \varepsilon)= & \varepsilon y_{i 1}\left(T_{0}, T_{1}, T_{2}, \ldots\right)+\varepsilon^{2} y_{i 2}\left(T_{0}, T_{1}, T_{2}, \ldots\right) \\
& +\cdots
\end{aligned}
$$

Substituting (41) and (42) into (35), according to the perturbation method, the each order equations of each order of $\varepsilon$ are established as follows:

$$
\varepsilon^{1}:
$$

$$
\begin{aligned}
& D_{0} y_{11}=\omega_{0} y_{21} \\
& D_{0} y_{21}=-\omega_{0} y_{11} \\
& D_{0} y_{31}=-\alpha_{3} y_{31} \\
& D_{0} y_{41}=-\alpha_{4} y_{41}
\end{aligned}
$$

$\varepsilon^{2}$

$$
\begin{aligned}
& D_{0} y_{12}=\omega_{0} y_{22}-D_{1} y_{11}+h_{12}\left(y_{1}\right) \\
& D_{0} y_{22}=-\omega_{0} y_{12}-D_{1} y_{21}+h_{22}\left(y_{1}\right) \\
& D_{0} y_{32}=-\alpha_{3} y_{32}+h_{32}\left(y_{1}\right) \\
& D_{0} y_{42}=-\alpha_{4} y_{42}+h_{42}\left(y_{1}\right)
\end{aligned}
$$

$$
\begin{aligned}
& \varepsilon^{3}: \\
& D_{0} y_{13}=\omega_{0} y_{23}-D_{1} y_{12}-D_{2} y_{11}+h_{13}\left(y_{1,2}\right) \\
& D_{0} y_{23}=-\omega_{0} y_{23}-D_{1} y_{22}-D_{2} y_{21}+h_{23}\left(y_{1,2}\right) \\
& D_{0} y_{33}=-\alpha_{3} y_{33}+h_{33}\left(y_{1,2}\right) \\
& D_{0} y_{43}=-\alpha_{4} y_{43}+h_{43}\left(y_{1,2}\right)
\end{aligned}
$$

In order to obtain the first-order approximate solution of system (34), according to the relationship between the two equations in (43), the following can be obtained:

$$
D_{0}^{2} y_{11}+\omega_{0}^{2} y_{11}=0
$$

The first-order approximate solution of $y_{1}$ can be solved as follows:

$$
\begin{aligned}
y_{11} & =r\left(T_{1}, T_{2}, \ldots\right) \cos \left[\omega_{0} T_{0}+\varphi_{1}\left(T_{1}, T_{2}, \ldots\right)\right] \\
& =R_{1}\left(T_{1}, T_{2}, \ldots\right) e^{j \omega_{0} T_{0}}+c c
\end{aligned}
$$

In (53), $R_{1}\left(T_{1}, T_{2}, \ldots\right)=\left(r\left(T_{1}, T_{2}, \ldots\right) / 2\right) e^{j \varphi\left(T_{1}, T_{2}, \ldots\right)}$.

$r$ and $\varphi$ represent the amplitude and phase of the first-order approximate periodic solution of $y_{1}$, respectively.
Similarly, the first-order approximate solution of $y_{2}$ can be expressed as follows:

$$
y_{21}=-r\left(T_{1}, T_{2}, \ldots\right) \sin \left[\omega_{0} T_{0}+\varphi\left(T_{1}, T_{2}, \ldots\right)\right]
$$

The asymptotically stable solutions $y_{j 1}=0, j=3,4$, which can be found clearly by (44) and (45) in the $\varepsilon^{1}$ term.

The relationship between the two equations in (46) is used to develop the second-order approximate solution for $y$ as follows:

$$
\begin{aligned}
D_{0}^{2} y_{12}+\omega_{0}^{2} y_{12}= & -D_{0} D_{1} y_{11}-\omega_{0} D_{1} y_{21}+D_{0} h_{12} \\
& +\omega_{0} h_{22}
\end{aligned}
$$

The second-order approximate solution of $\mathrm{y}$ can be expressed as follows:

$$
y_{i 2}=\sum_{n=0}^{2}\left[E_{i} \cos (n \theta)+F_{i} \sin (n \theta)\right]
$$

Substituting $y_{i 1}$ and $y_{i 2}$ into (55), $\mathrm{n}=1,2$, the expressions for the trigonometric functions $\cos (n \theta)$ and $\sin (n \theta), \mathrm{n}=1,2,3$, can be obtained. Eliminating system secular terms, the second-order approximate solution of $y$ and the $D_{1} r, D_{1} \varphi$ can be obtained.

Similarly, according to the relationship between the two equations in (49), the equation can be rewritten as follows:

$$
\begin{aligned}
D_{0}^{2} y_{13}+\omega_{0}^{2} y_{13}= & -D_{0} D_{2} y_{11}-\omega_{0} D_{2} y_{21}-D_{0} D_{1} y_{12} \\
& -\omega_{0} D_{1} y_{22}+D_{0} h_{13}\left(y_{1,2}\right) \\
& +\omega_{0} h_{23}\left(y_{1,2}\right)
\end{aligned}
$$

Similarly, the third-order approximate solution of $y$ can be expressed as follows:

$$
y_{i 3}=\sum_{n=0}^{3}\left[P_{i} \cos (n \theta)+Q_{i} \sin (n \theta)\right]
$$

The third-order approximate solution of $y$ and the $D_{2} r$, $D_{2} \varphi$ can be achieved through the harmonic balance method.

According to $D_{0} r, D_{1} r, D_{2} r$ and $D_{0} \varphi, D_{1} \varphi, D_{2} \varphi$ obtained from the synthesis of nonlinear vibration theory, the third-order normal form in the polar coordinate of system can be obtained.

$$
\begin{aligned}
\dot{r} & =D_{0} r+D_{1} r+D_{2} r \\
& =-3.3722 \eta r+\left(-0.3277 K_{n}+0.0029\right) r^{3} \\
\dot{\theta} & =\omega_{c}+D_{0} \varphi+D_{1} \varphi+D_{2} \varphi \\
& =6.1268+4.0868 \eta+\left(0.2001 K_{n}+0.00059\right) r^{2}
\end{aligned}
$$

The opening item in (59) is shown as follows:

$$
\begin{aligned}
& \delta_{r}=\frac{1}{2}\left(\frac{\partial^{2} \dot{y}_{1}}{\partial y_{1} \partial \eta}+\frac{\partial^{2} \dot{y}_{2}}{\partial y_{2} \partial \eta}\right)=-3.3722 \\
& \delta_{\theta}=\frac{1}{2}\left(\frac{\partial^{2} \dot{y}_{1}}{\partial y_{2} \partial \eta}-\frac{\partial^{2} \dot{y}_{2}}{\partial y_{1} \partial \eta}\right)=4.0868
\end{aligned}
$$




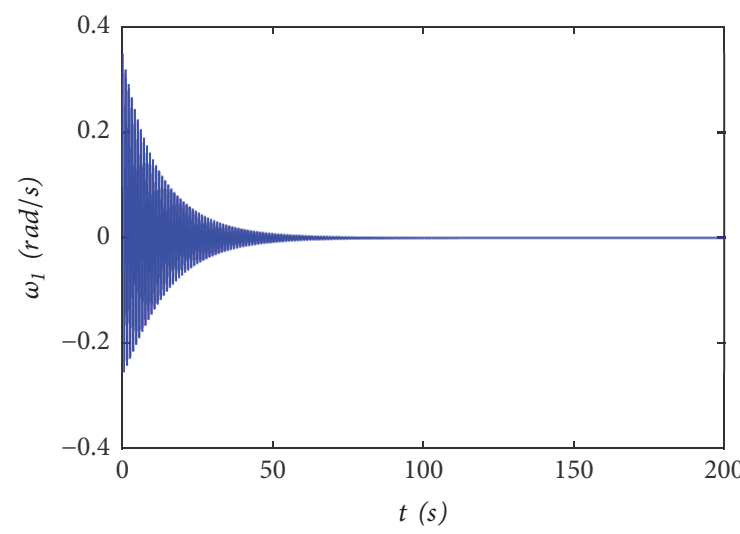

(a) diagram.

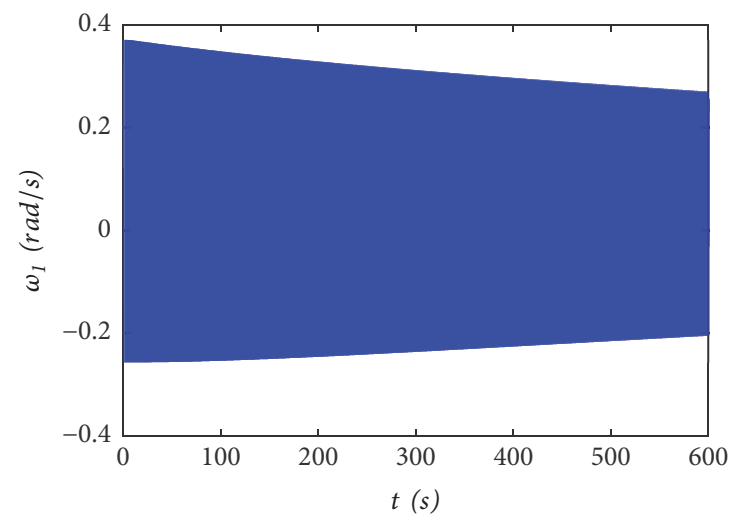

(a)

FIGURE 8: When $R=0.0566 \Omega, K_{\mathrm{c}}=1.5$, and $K_{\mathrm{n}}=0$, initial value $=\left[\begin{array}{llll}0.1 & 0.1 & 0.1 & 0.1\end{array}\right]$, system motion status: (a) time history diagram; (b) phase diagram.

The necessary and sufficient condition for the occurrence of supercritical Hopf bifurcation in the system is listed as follows:

$$
-0.3277 K_{n}+0.0029<0
$$

That is,

$$
\begin{aligned}
K_{n} & >0.0088 \\
r & =\sqrt{\frac{-3.3722 \eta}{0.3277 K_{n}-0.0029}}
\end{aligned}
$$

The numerical simulation for the limit cycle which is produced in the neighborhood of the Hopf bifurcation point of the controlled system verifies the effectiveness of the designed linear feedback controller and nonlinear controller on the Hopf bifurcation transmission, the limit cycle stability, and amplitude adjustment. Taking the linear controller control parameter $K_{\mathrm{c}}=1.5$ and the bifurcation parameter $R=0.085 \Omega$

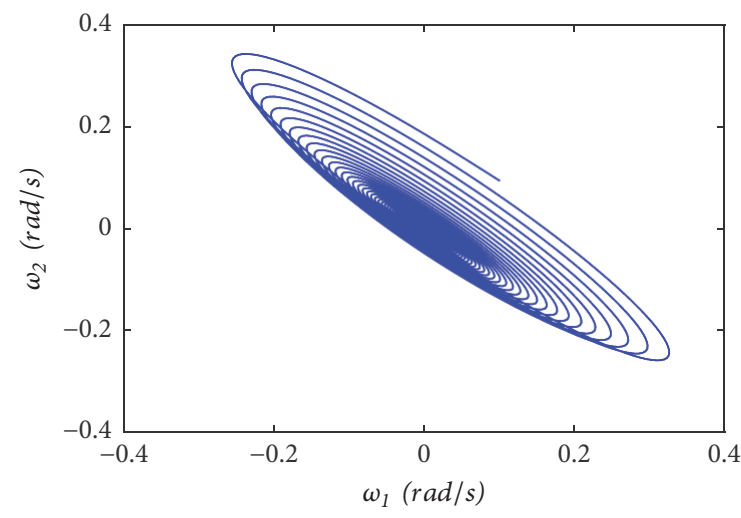

(b)

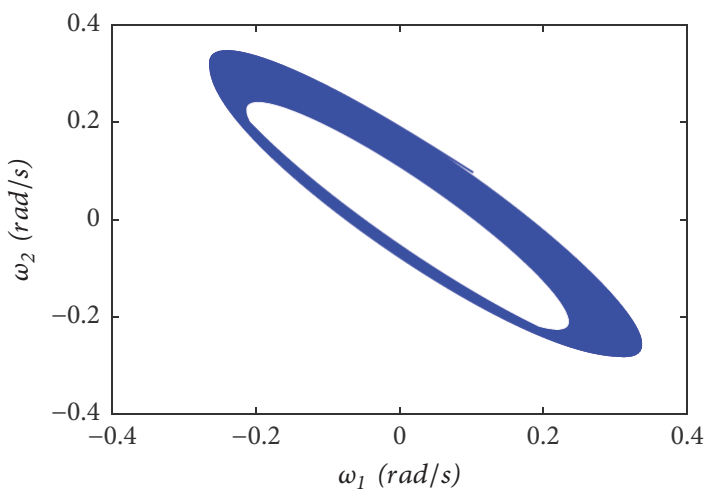

(b)

as an example, the system time history diagram and phase diagram are drawn as follows when there is no nonlinear feedback controller installed.

Comparing Figure 6 with Figure 7, it can be found that, after adding the linear controller and the drive motor resistance $R=0.085$, the original system runs from chaotic into a smooth state at the equilibrium point, supporting the idea that the linear controller could effectively expand the stable interval in the system.

When the nonlinear controller is not added, the subcritical Hopf bifurcation still occurs near the bifurcation point of the original system. Taking $R=0.0566 \Omega$ as an example, when the initial value takes the smaller value $\left[\begin{array}{llll}0.1 & 0.1 & 0.1 & 0.1\end{array}\right]$, the system works in steady state. With other parameters unchanged, Hopf bifurcation occurs in the system when the initial value is increased to $\left[\begin{array}{llll}0.7 & 0.7 & 0.7 & 0.7\end{array}\right]$. The results of the numerical simulation are shown in Figures 8 and 9.

Without adding a nonlinear controller, the system subcritical Hopf bifurcation still occurs at the point of bifurcation. The system will produce instability and oscillation, 


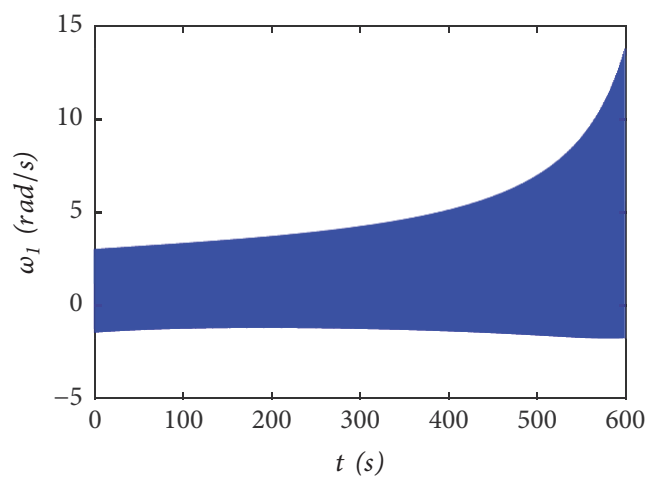

(a)

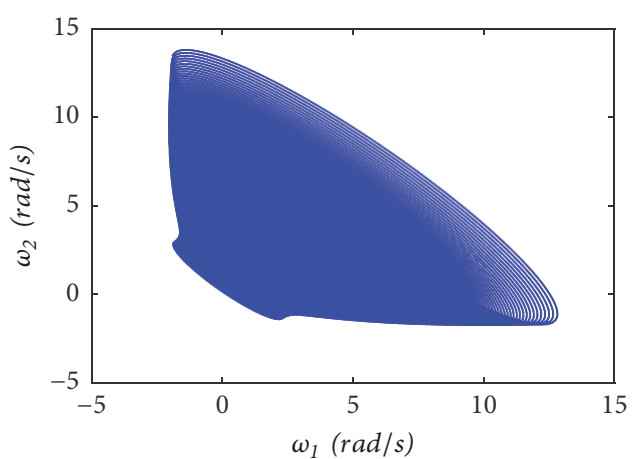

(b)

Figure 9: When $R=0.0566 \Omega, K_{\mathrm{c}}=1.5$, and $K_{\mathrm{n}}=0$, initial value $=\left[\begin{array}{llll}0.7 & 0.7 & 0.7 & 0.7\end{array}\right]$, system motion status: (a) time history diagram; (b) phase diagram.

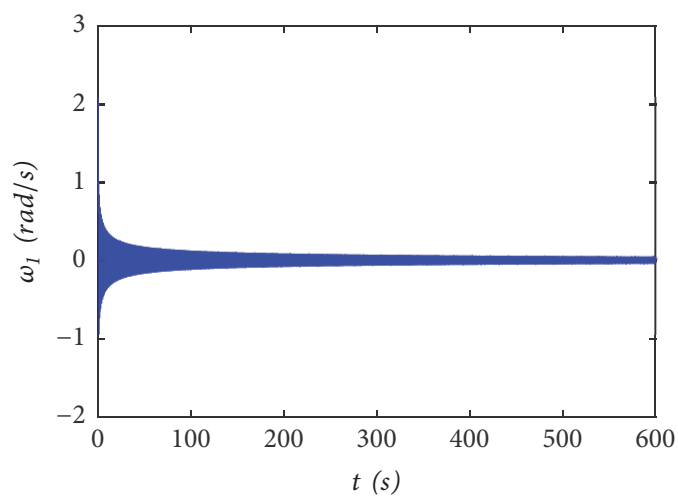

(a)

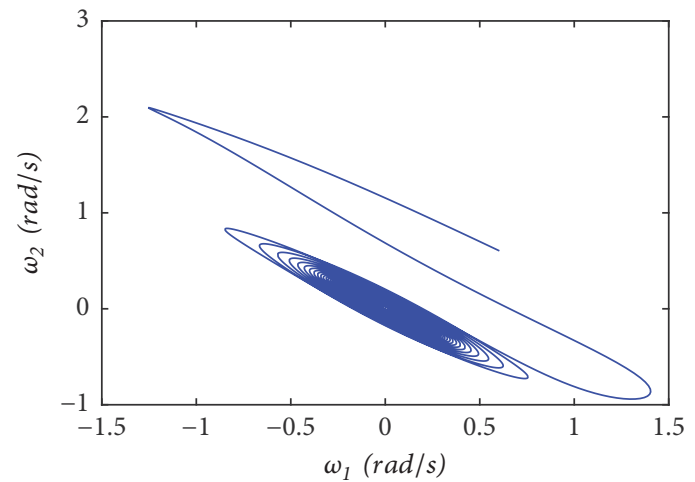

(b)

FIGURE 10: When $R=0.0566 \Omega, K_{\mathrm{c}}=1.5$, and $K_{\mathrm{n}}=0.5$, initial value $=\left[\begin{array}{llll}0.7 & 0.7 & 0.7 & 0.7\end{array}\right]$, system motion status: (a) time history diagram; (b) phase diagram.

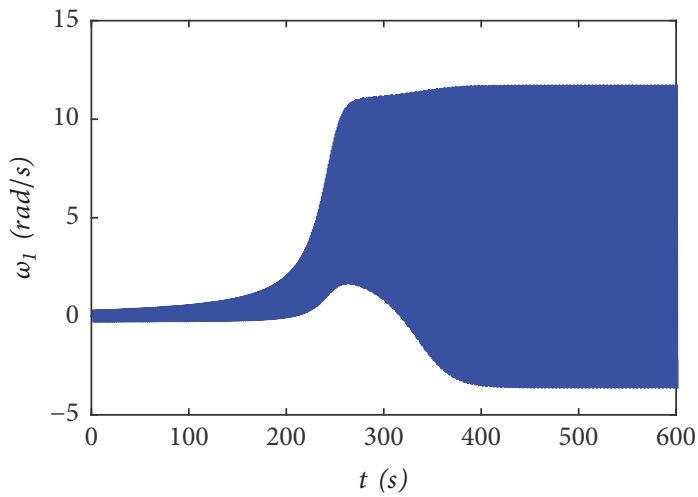

(a)

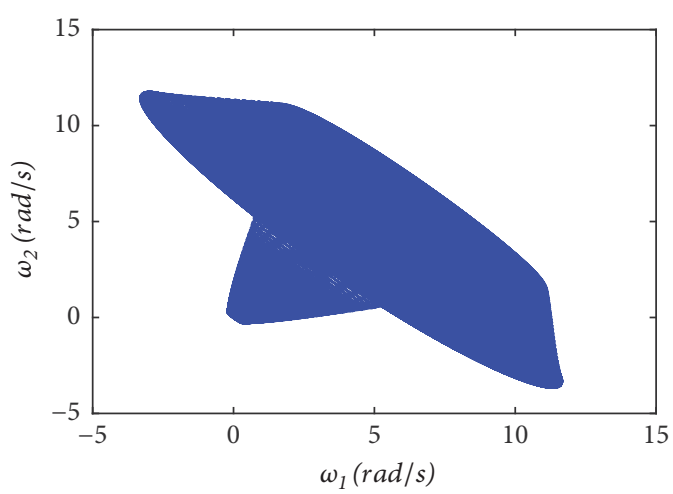

(b)

FIGURE 11: When $R=0.05 \Omega, K_{\mathrm{c}}=1.5$, and $K_{\mathrm{n}}=0.5$, initial value $=\left[\begin{array}{llll}0.1 & 0.1 & 0.1 & 0.1\end{array}\right]$, system motion status: (a) time history diagram; (b) phase diagram.

which will damage the system, affect the system work efficiency, and even cause security accidents. Therefore, it is necessary to install nonlinear controller. Taking nonlinear controller parameter $K_{\mathrm{n}}=0.5$, motor resistance $R=0.0566 \Omega$, and initial value $=\left[\begin{array}{llll}0.7 & 0.7 & 0.7 & 0.7\end{array}\right]$, numerical simulation results are shown in Figure 10.

From Figure 10, it is suggested that, with the nonlinear controller installed, the system operates from unstable state 


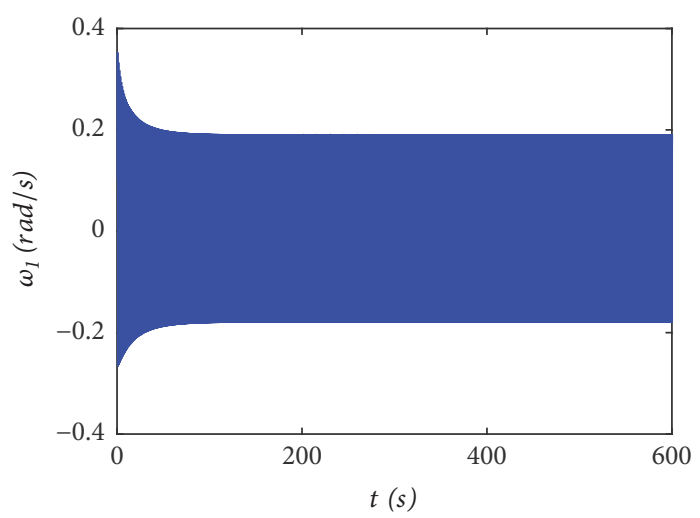

(a)

FIGURE 12: When $R=0.05 \Omega, K_{\mathrm{c}}=1.5$, and $K_{\mathrm{n}}=10$, initial value $=\left[\begin{array}{llll}0.1 & 0.1 & 0.1 & 0.1\end{array}\right]$, system motion status: (a) time history diagram; (b) phase diagram.

into a stable state when motor resistance $R=0.0566 \Omega$ and initial value $=\left[\begin{array}{llll}0.7 & 0.7 & 0.7 & 0.7\end{array}\right]$, indicating that the designed nonlinear controller has a sound control effect.

When the nonlinear controller is added to the controlled system, the system supercritical Hopf bifurcation is generated at the neighborhood of the Hopf bifurcation point $R_{0}^{*}$. When the bifurcation parameter $R=0.05 \Omega<R_{0}^{*}$, the stable limit cycle is generated near the equilibrium point, as shown in Figures 11 and 12. It effectively avoids the more harmful subcritical destabilizing oscillations with greater harm. When $R$ is the same, the amplitude of the stable limit cycle can be controlled by changing the parameter $K_{n}$. Comparing Figure 11 with Figure 12, we can see that when $K_{n}=10$, the system limit cycle amplitude is obviously smaller than it when $K_{\mathrm{n}}=0.05$. Also as the $K_{\mathrm{n}}$ increases, the limit cycle amplitude of the system decreases. Although there is a certain error between the limit cycle amplitude obtained by numerical simulation and the analytical calculation in Figures 11 and 12, the variation trend of the limit cycle amplitude is consistent. Therefore, the amplitude of the system oscillation can be reduced by increasing the feedback coefficient $K_{n}$. The damage degree of the torsional vibration of the semidirect drive transmission system in coal cutter is reduced.

\section{Conclusions}

As the three-phase asynchronous motor has been replaced by the PMSM, in which the intermediate drive devices are shortened, the permanent magnet semidirect drive cutting transmission system of coal cutters has been composed, minimizing the breakdown rate and improving the system stable performance greatly. When the temperature changes or the working conditions become worse, its unstable vibration may appear caused by resistance value of PMSM. On the basis of this and with its complex electromechanical coupling characteristics combined, the dynamics equation of nonlinear torsional vibration system is set up, analyzing the Hopf bifurcation both qualitatively and quantitatively. In addition, given the q-axis as the controlling variable, the nonlinear

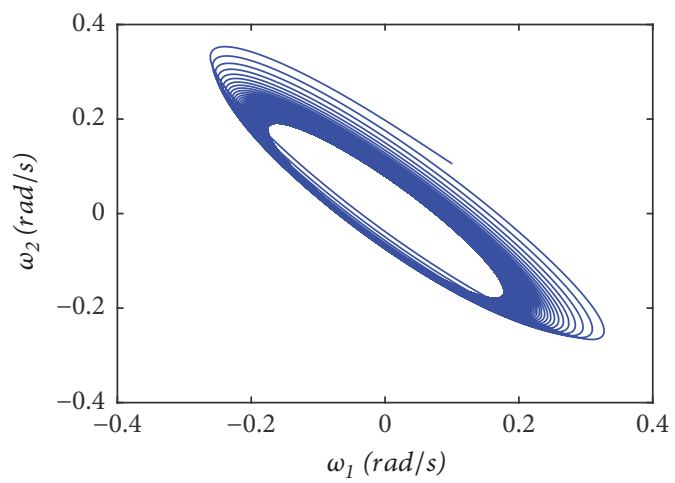

(b) speed feedback controller is presented, eliminating the torsional vibration effectively. The results are demonstrated as follows:

(1) Coal cutters perform in a relatively adverse environment, which may bring a change in electrical parameters. As a result, subcritical Hopf bifurcation may occur in the main driving system, causing the system extremely vibration which may exert a negative effect on the system performance.

(2) The control effect of the nonlinear controller is verified by numerical simulation. On the one hand, the linear part can expand the stable region of the system. On the other hand, the nonlinear part can reduce subcritical bifurcation phenomenon as well as the limit cycle amplitudes.

(3) The amplitudes of a stable limit cycle decrease along with the increase of the nonlinear parameters. Since the proposed controller may implement effective control on the torsional vibration in the scraper conveyor of main power system electrically, a safe and reliable guarantee is provided for the application of high-power permanent magnet synchronous motors, an expectation of the utilizing in the area of large-scale mechanical equipment.

\section{Data Availability}

The data used to support the findings of this study are available from the corresponding author upon request after publication of this article.

\section{Conflicts of Interest}

The authors declare no conflicts of interest.

\section{Acknowledgments}

This work is supported by the National Natural Science Foundation of China (Grant no. 51775543), the Key Research and Development Project of Xuzhou (Grant no. KC17014), 
and the Project Funded by the Priority Academic Program Development of Jiangsu Higher Education Institutions (PAPD).

\section{References}

[1] N. Bilgin, M. A. Demircin, H. Copur, C. Balci, H. Tuncdemir, and N. Akcin, "Dominant rock properties affecting the performance of conical picks and the comparison of some experimental and theoretical results," International Journal of Rock Mechanics \& Mining Sciences, vol. 43, no. 1, pp. 139-156, 2006.

[2] C. Zhu, X. Lu, Z. Gao, F. Yan, C. Guo, and X. Zhang, "Effect of high-voltage thermal breakdown on pore characteristics of coal," International Journal of Mining Science and Technology, vol. 27, no. 6, pp. 1051-1055, 2017.

[3] L. Sheng, W. Li, Y. Wang, M. Fan, and X. Yang, "Sensorless control of a shearer short-range cutting interior permanent magnet synchronous motor based on a new sliding mode observer," IEEE Access, vol. 5, no. 99, pp. 18439-18450, 2017.

[4] W. Tao, C. Chen, H. Jun, and R. Ting, "Effect of bolt rib spacing on load transfer mechanism," International Journal of Mining Science and Technology, vol. 27, no. 3, pp. 431-434, 2017.

[5] Q. Wei and X.-Y. Wang, "Chaos controlling of permanent magnet synchronous motor base on dither signal," Journal of Vibration and Control, vol. 19, no. 16, pp. 2541-2550, 2013.

[6] K. Liu and Z. Q. Zhu, "Online estimation of the rotor flux linkage and voltage-source inverter nonlinearity in permanent magnet synchronous machine drives," IEEE Transactions on Power Electronics, vol. 29, no. 1, pp. 418-427, 2014.

[7] X. Chen, S. Yuan, and Z. Peng, "Nonlinear vibration for PMSM used in HEV considering mechanical and magnetic coupling effects," Nonlinear Dynamics, vol. 80, no. 1-2, pp. 541-552, 2015.

[8] H.-J. Shin, J.-Y. Choi, H.-I. Park, and S.-M. Jang, "Vibration analysis and measurements through prediction of electromagnetic vibration sources of permanent magnet synchronous motor based on analytical magnetic field calculations," IEEE Transactions on Magnetics, vol. 48, no. 11, pp. 4216-4219, 2012.

[9] R. Shanmugasundram, K. M. Zakaraiah, and N. Yadaiah, "Effect of parameter variations on the performance of direct current (DC) servomotor drives," Journal of Vibration and Control, vol. 19, no. 10, pp. 1575-1586, 2013.

[10] G. W. Li, Dynamic characteristics analysis of shearer electrohydraulic short-range cutting transmission system under dynamic excitation [Master thesis], Chongqing University, China, 2016.

[11] J. Li and H. Guo, "Vibration characteristics of shearer cutting unit based on multibody dynamics," Electronic Journal of Geotechnical Engineering, vol. 19, no. 4, pp. 3195-3208, 2014.

[12] L. J. Zhao and J. B. Lan, "Dynamics simulation of driving system of shearer cutting machine," Journal of vibration. and Shock, vol. 33, pp. 106-110, 2014.

[13] Z. Li and Z. Peng, "Nonlinear dynamic response of a multidegree of freedom gear system dynamic model coupled with tooth surface characters: a case study on coal cutters," Nonlinear Dynamics, vol. 84, no. 1, pp. 271-286, 2016.

[14] Y. Jiang, H. Zhu, Z. Li, and Z. Peng, "The nonlinear dynamics response of cracked gear system in a coal cutter taking environmental multi-frequency excitation forces into consideration," Nonlinear Dynamics, vol. 84, no. 1, pp. 203-222, 2016.

[15] C. Liu, D. Qin, and Y. Liao, "Electromechanical dynamic analysis for the drum driving system of the long-wall shearer,"
Advances in Mechanical Engineering, vol. 7, no. 10, pp. 202-208, 2015.

[16] N. Feki, G. Clerc, and P. Velex, "An integrated electromechanical model of motor-gear units - Applications to tooth fault detection by electric measurements," Mechanical Systems and Signal Processing, vol. 29, pp. 377-390, 2012.

[17] A. M. Knight and S. P. Bertani, "Mechanical fault detection in a medium-sized induction motor using stator current monitoring," IEEE Transactions on Energy Conversion, vol. 20, no. 4, pp. 753-760, 2005.

[18] S. H. Kia, H. Henao, and G.-A. Capolino, "Torsional vibration assessment using induction machine electromagnetic torque estimation," IEEE Transactions on Industrial Electronics, vol. 57, no. 1, pp. 209-219, 2010.

[19] X. Tang, W. Yang, X. Hu, and D. Zhang, "A novel simplified model for torsional vibration analysis of a series-parallel hybrid electric vehicle," Mechanical Systems and Signal Processing, vol. 85, pp. 329-338, 2017.

[20] S. Liu, S. Zhao, B. Niu, J. Li, and H. Li, "Stability analysis of a nonlinear electromechanical coupling transmission system with time delay feedback," Nonlinear Dynamics, vol. 86, no. 3, pp. 1863-1874, 2016.

[21] S. Liu, S. Zhao, Z. Wang, and H. Li, "Stability and Hopf bifurcation of a nonlinear electromechanical coupling system with time delay feedback," Chinese Physics B, vol. 24, no. 1, pp. 345-353, 2015.

[22] W. Bartelmus, "Mathematical modelling and computer simulations as an aid to gearbox diagnostics," Mechanical Systems and Signal Processing, vol. 15, no. 5, pp. 855-871, 2001.

[23] M. T. Khabou, N. Bouchaala, F. Chaari, T. Fakhfakh, and M. Haddar, "Study of a spur gear dynamic behavior in transient regime," Mechanical Systems and Signal Processing, vol. 25, no. 8, pp. 3089-3101, 2011.

[24] D. Yu, Y. Liu, G. Wang, L. Cai, and J. Qiu, "Low frequency torsional vibration gaps in the shaft with locally resonant structures," Physics Letters A, vol. 348, no. 3-6, pp. 410-415, 2006.

[25] F. Chaari, M. S. Abbes, F. V. Rueda, A. F. del Rincon, and M. Haddar, "Analysis of planetary gear transmission in nonstationary operations," Frontiers of Mechanical Engineering, vol. 8, no. 1, pp. 88-94, 2013.

[26] P. Shi, B. Liu, and D. Hou, "Global dynamic characteristic of nonlinear torsional vibration system under harmonically excitation," Chinese Journal of Mechanical Engineering, vol. 22, no. 1, pp. 132-139, 2009.

[27] P.-M. Shi, D.-Y. Han, and B. Liu, "Chaos and chaotic control in a relative rotation nonlinear dynamical system under parametric excitation," Chinese Physics B, vol. 19, no. 9, Article ID 090306, pp. 112-117, 2010.

[28] L. Xu and Y. Gao, "Bifurcation and chaotic vibration in electromechanical integrated toroidal drive," Journal of Vibration and Control, vol. 21, no. 8, pp. 1556-1565, 2015.

[29] T. Szolc, R. Konowrocki, M. Michajłow, and A. Pręgowska, "An investigation of the dynamic electromechanical coupling effects in machine drive systems driven by asynchronous motors," Mechanical Systems and Signal Processing, vol. 49, no. 1-2, pp. 118-134, 2014.

[30] B. Liu, S. Liu, Y. Zhang, and Y. Wen, "Bifurcation control for electromechanical coupling vibration in rolling mill drive system based on nonlinear feedback," Journal of Mechanical Engineering, vol. 46, no. 8, pp. 160-166, 2010. 


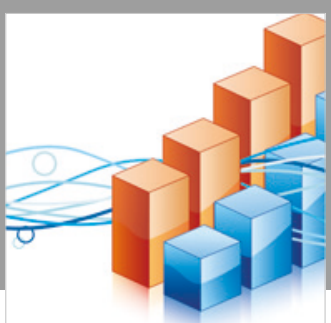

Advances in

Operations Research

\section{-n-m}
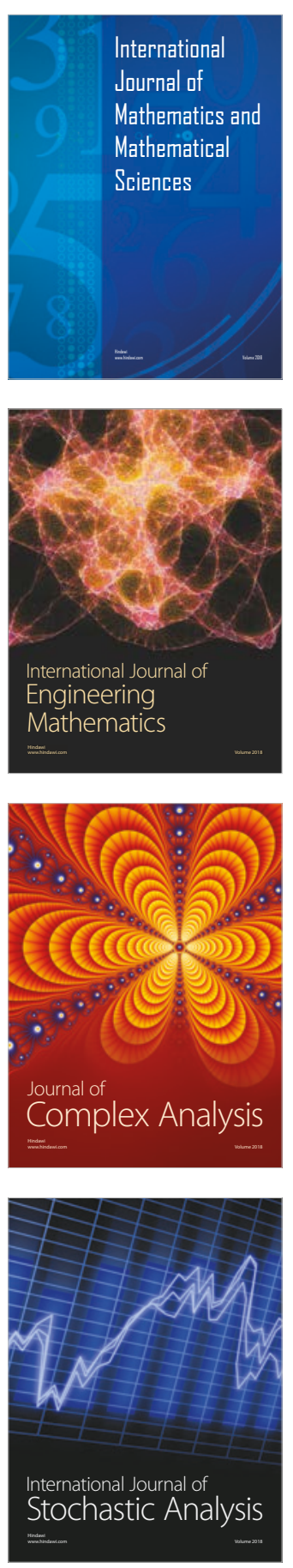
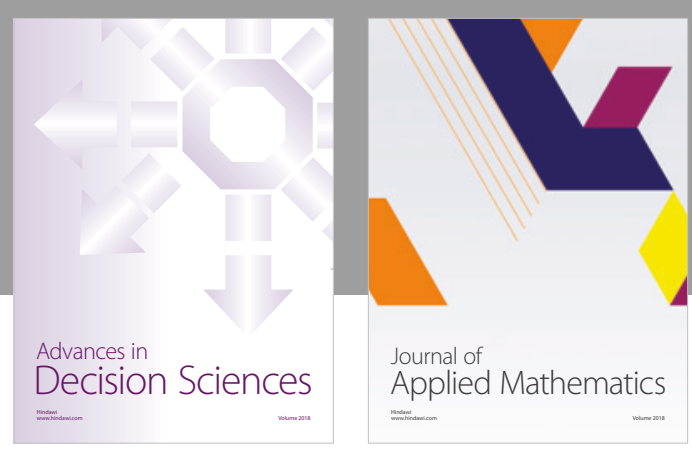

Journal of

Applied Mathematics
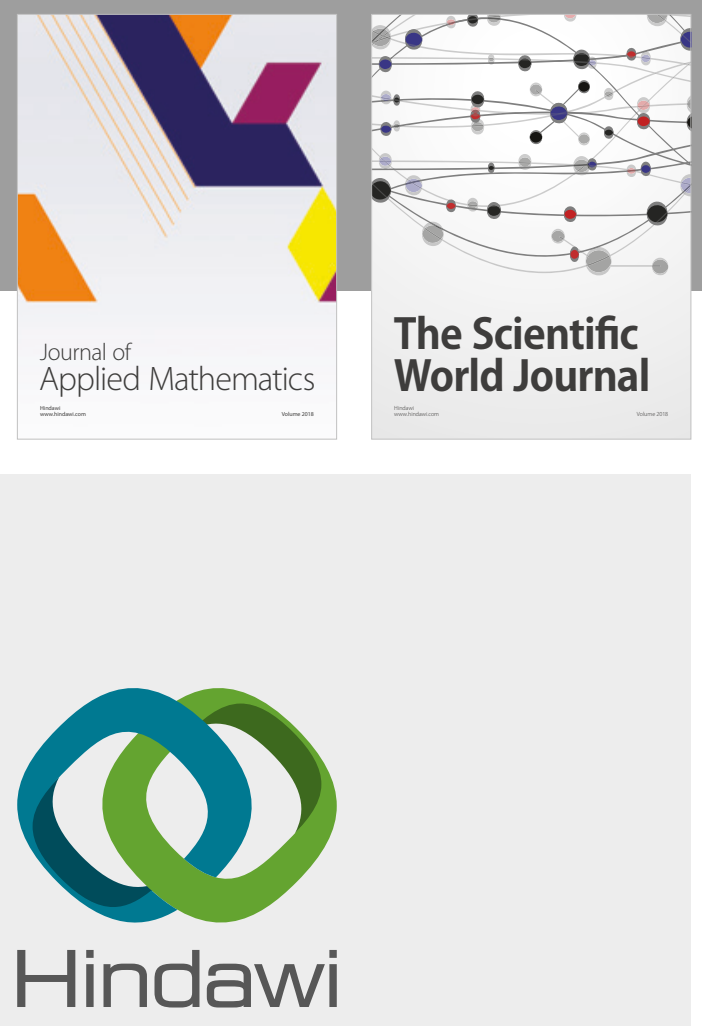

Submit your manuscripts at

www.hindawi.com

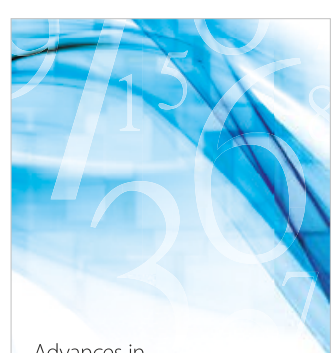

Advances in
Numerical Analysis
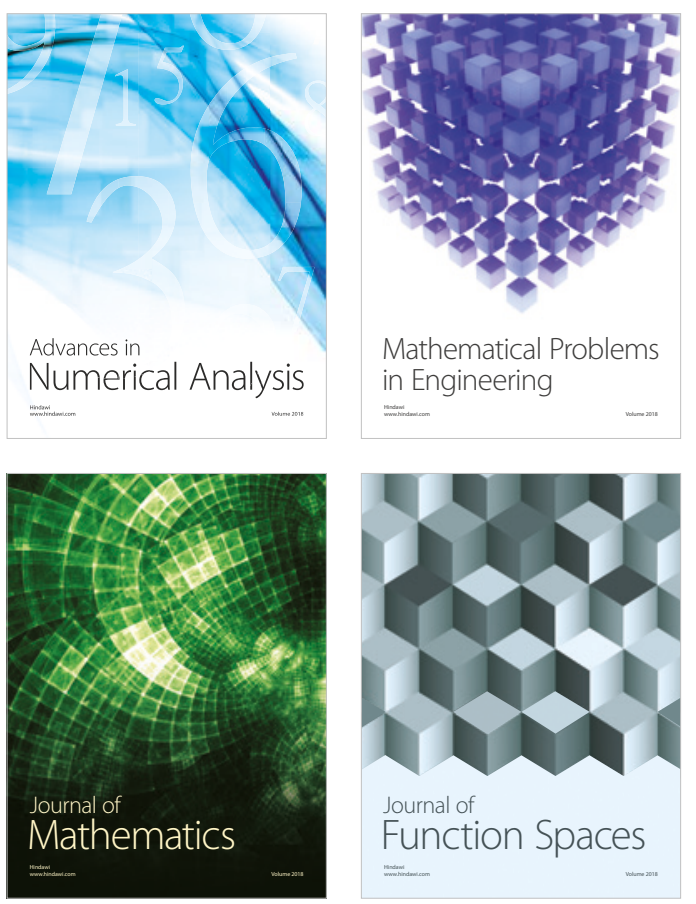

Mathematical Problems in Engineering

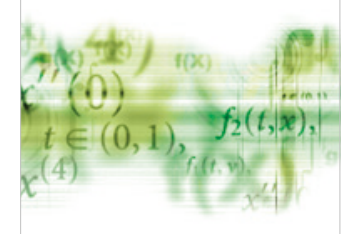

International Journal of

Differential Equations

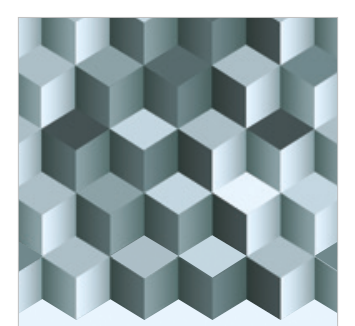

Journal of

Function Spaces
The Scientific

World Journal

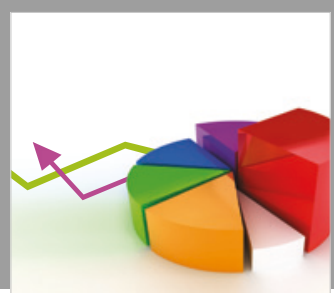

Journal of

Probability and Statistics
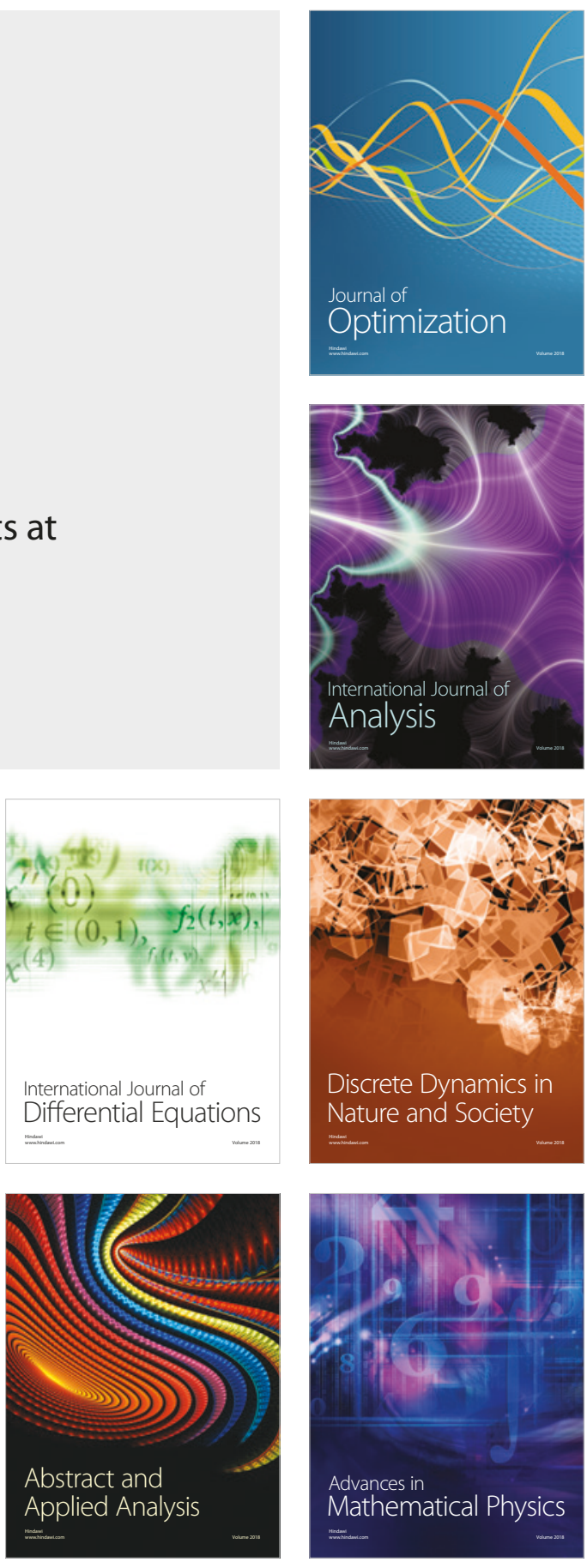